\title{
Delayed Neutrons Energy Spectrum Flux Profile of Nuclear Materials in Ghana's Miniature Neutron Source Reactor Core
}

\author{
R. B. M. Sogbadji ${ }^{2 *}$, R. G. Abrefah ${ }^{2}$, E. Ampomah-Amoako ${ }^{1}$, S. A. Birikorang ${ }^{2}$, S. E. Agbemava ${ }^{2}$, \\ B. J. B. Nyarko ${ }^{2}$, H. C. Odoi ${ }^{1}$ \\ ${ }^{1}$ Department of Nuclear Engineering, University of Ghana, School of Nuclear and Allied Science, Atomic Energy, \\ Accra, Ghana \\ ${ }^{2}$ Ghana Atomic Energy Commission, National Nuclear Research Institute, Legon, Ghana. \\ E-mail: robertmawuko@yahoo.com \\ Received April 23, 2011; revised May 24, 2011; accepted June 27, 2011
}

\begin{abstract}
A slightly prompt critical nuclear reactor would increase the neutron flux exponentially at a high rate causing the reactor to become uncontrollable, however due to the delayed neutrons, it is possible to leave the reactor in a subcritical state as far as only prompt neutrons are concerned and to also sustain the chain reaction when it is going to die out. The delay neutron flux spectrum of the compact core of the Ghana's miniature neutron source reactor (MNSR) was studied using the Monte Carlo method. 20,484 energy groups combined for all three categories of the energy distribution, thermal, slowing down and fast regions were modeled to create small energy bins. The moderator, the inner irradiation channels, the annulus beryllium reflector and the outer irradiation channels were the regions monitored. The delay thermal neutrons recorded its highest flux in the inner irradiation channel with an average flux of $(4.01270 .0076) \times 1008 \mathrm{n} / \mathrm{cm}^{2} \cdot \mathrm{s}$, followed by the outer irradiation channel with an average flux of $(2.45240 .0049) \times 1008 \mathrm{n} / \mathrm{cm}^{2} \cdot \mathrm{s}$. The beryllium reflector recorded the lowest flux in the thermal region. These values of the thermal energy range occurred in the energy range $(0-0.625 \times 10-07) \mathrm{MeV}$. The inner irradiation channel again recorded the highest average flux of $(1.2050 \pm 0.0501) \times 1007 \mathrm{n} / \mathrm{cm}^{2} \cdot \mathrm{s}$ at the slowing down region in the energy range $(0.821-6.94) \mathrm{MeV}$. The outer irradiation channel recorded the lowest flux in this region. In the fast energy region, $(6.96-20)$ $\mathrm{MeV}$, the core, where the moderator is found, the same trend was observed with the inner irradiation channel recording the highest flux at an average flux of $(2.0647 \pm 0.3260) \times 1006 \mathrm{n} / \mathrm{cm}^{2} \cdot \mathrm{s}$.The outer irradiation channel recorded the second highest flux while the annulus beryllium reflector recorded very low flux in this region. The final k-effective contribution from only delay neutrons is 0.00834 with the delay neutron fraction being $0.01357 \pm 0.00049$, hence the Ghana MNSR has good safety inherent feature.
\end{abstract}

Keywords: Radioactivity, Doses, Water, Gamma Spectroscopy, Oil Areas, Nigeria

\section{Introduction}

Safety is key in the design of nuclear reactors, if a nuclear reactor happened to be prompt critical—even very slightly - the number of neutrons would increase exponentially at a high rate, and very quickly the reactor would become uncontrollable by means of cybernetics. The control of the power rise would then be left to its intrinsic physical stability factors, like the thermal dilatation of the core, or the increased resonance absorptions of neutrons, that usually tend to decrease the reactor's reactivity when temperature rises; but the reactor would run the risk of being damaged or destroyed by heat [1].

However, thanks to the delayed neutrons, it is possible to leave the reactor in a subcritical state as far as only prompt neutrons are concerned: the delayed neutrons come a moment later, just in time to sustain the chain reaction when it is going to die out. In that regime, neutron production overall still grows exponentially, but on a time scale that is governed by the delayed neutron production, which is slow enough to be controlled. Thus, by widening the margins of non-operation and supercriticality and allowing more time 
to regulate the reactor, the delayed neutrons are essential to inherent reactor safety and even in reactors requiring active control [1].

To design a nuclear reactor properly, it is necessary to predict how the neutrons will be distributed throughout the system. Unfortunately, determining the neutron distribution is a difficult problem in general. The neutrons in a nuclear reactor move in a complicated path as result of repeated nuclear collisions. To a first approximation, however, the overall effect of these collisions is that the neutrons undergo a kind of diffusion in the reactor medium, much like the diffusion of one gas in another. Since neutrons in a nuclear reactor actually have a distribution in energy, this distribution must be accounted for in the diffusion equation. Neutrons are emitted in fission with a continuous energy spectrum and this distribution broadens as the neutrons are scattered in the medium and diffuse about the system, losing energy in elastic and inelastic collisions.

\section{Design Considerations of Ghana Research Reactor-1}

A detailed description of the operating characteristics of Ghana's MNSR has been presented elsewhere [2]. Table 1 shows the design specifications of Ghana research reactor-1. Due to its inherent safety features, stability of flux and moderate cost, the MNSR has recently found

Table 1. Technical specifications of Ghana's MNSR.

\begin{tabular}{cc}
\hline PARAMETER & DESCRIPTION \\
\hline Reactor design type & Tank-in-pool \\
Rated thermal power & $30 \mathrm{~kW}$ \\
Excess reactivity & $3.5-4.0 \mathrm{mk}$ \\
Fuel & U-Al dispersed in Al \\
Enrichment & $90.2 \%, \mathrm{HEU}$ \\
Diameter of fuel meat & $4.3 \mathrm{~mm}$ \\
Diameter of fuel element & $5.5 \mathrm{~mm}$ \\
No. of fuel elements & 344 \\
U-235 loading & $\sim 1 \mathrm{~kg}$ \\
Core diameter & $23.1 \mathrm{~cm}$ \\
Core height (active) & $23.0 \mathrm{~cm}$ \\
No. of irradiation channels & 10 \\
Inner channels & $5.5 \mathrm{~mm}$ \\
Flux in inner channel (peak) & $1.0 \mathrm{E}+12 \mathrm{n} / \mathrm{cm}^{2} . \mathrm{s}$ \\
Flux in outer channel (peak) & $5.0 \mathrm{E}+11 \mathrm{n} / \mathrm{cm}^{2} . \mathrm{s}$ \\
Reactor cooling mode & Natural convection \\
Height of inlet orifice & $6 \mathrm{~mm}$ \\
Height of outlet orifice & Beryllium metal alloy \\
\hline Reflector material & 7.50 control rod
\end{tabular}

enormous application in various fields of science [3], particularly in trace elements in matrices of biological and environmental samples [4] and soil fertility studies and geochemical mapping [5]. Figure 1 shows the cross-sectional view of the Ghana research reactor-1

\section{Theory and Method}

The approximate value of the neutron distribution can be found by solving the diffusion equation. This procedure, which is called the diffusion approximation, was used for the design of most of the early reactors. One of the most effective ways to calculate diffusion of neutrons is by the group-diffusions method but the complexity of the group-diffusion or the multigroup equations as they are sometimes called, it is common practice to use a computer program to evaluate the group fluxes. The techniques by which this is done involve approximating the derivatives by numerical methods and then requiring the equation hold only at a series of discrete points in space. The equations are then reduced to algebraic equations valid for only those points. The exact approaches vary for each computer program. In this work, the Monte Carlo method was used for the simulations.

In recent times the Monte Carlo approach has been included to reactor analysis. In particular, multipurpose Monte Carlo particle transport codes generally have the capability to model and treat different complicated geometries in 3-Dimensions and also simulate the transport behavior of different particles and nuclear interaction processes. Good and accurate modeling of the different zones and diverse geometries of the MNSR reactor is important for realizing good neutronics, particle transport

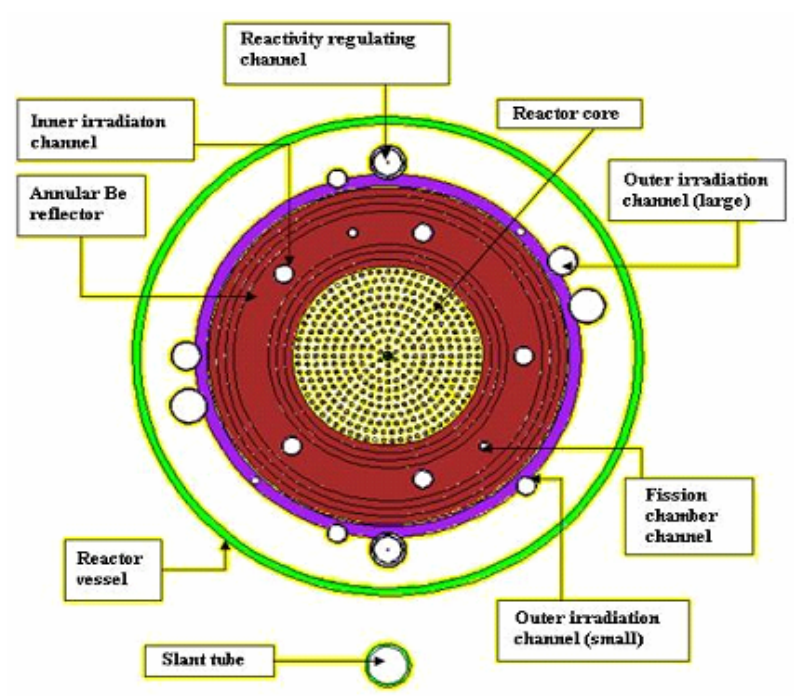

Figure.1 MCNP plot of GHARR-1 core configuration showing fuel region (reactor core), inner and outer irradiation channels and annulus beryllium reflector [6]. 
simulation, and physics analysis. For these reasons, theversatile and widely utilized MCNP code particle trans port code was employed to develop a 3-D Monte Carlo model for MNSR for particle transport simulation and neutronic analysis of MNSR reactors [4,7]. The 3-D GHARR-1 Monte Carlo model using the MCNP code was used to simulate some reactor physics parameters such as nuclear criticality and core reactivities of the GHARR-1 facility operating on 90.2\% HEU U-Al fuel. Neutron particle transport simulations were performed for a clean fresh core (zero burnup) at room temperature. Criticality calculations were performed to determine keff and neutron fluxes at specific energy bins.

\section{The Monte Carlo Approach}

MCNP simulations were made for 500,000 neutron particles and 400 criticality cycles, corresponding to 200 million particle histories.

An initial criticality guess of $k_{\text {eff }}=1.004$ was used in respect of the fact that the cores excess reactivity for the GHARR-1 HEU core is $3.99-4.00 \mathrm{mk}$. The MCNP criticality and neutronics simulations were normalized to the steady-state full power level of $30 \mathrm{~kW}$ corresponding to peak thermal neutron flux of $1.0 \times 10^{12} \mathrm{n} / \mathrm{cm}^{2} \cdot \mathrm{s}$ (critical operation mode).

The MCNP neutron energy spectrum was performed for 20,484 energy groups combined for all three categories of the energy distribution, thus thermal region, slowing down region and fast region. The spectrum of the total neutron flux was simulated and then that for the prompt neutron spectrum was simulated, the difference between these two simulations produced the delayed neutron energy spectrum.

Delayed neutron flux=Total neutron flux - Prompt neutron flux

The following energy bins were used in the MCNP tally for the various energy groups; $1.89 \mathrm{e}-8 \mathrm{MeV}$ energy bin for $(0-6.25 \mathrm{e}-7) \mathrm{MeV}$ thermal energy range, 1.89e-3 energy bin for (8.21e-1 - 6.94) $\mathrm{MeV}$ slowing down energy range and 1.89e-3MeV energy bin for (6.96 - 20) MeV fast energy range.

Delayed neutrons are associated with the beta decay of the fission products. After prompt fission neutron emission, the residual fragments are still neutron rich and undergo a beta decay chain. The more neutron rich the fragment, the more energetic and faster the beta decay. In some cases the available energy in the beta decay is high enough to leave the residual nucleus in such a highly excited state that neutron emission instead of gamma emission occurs.

The delay neutron fraction (DNF) is given as:

$$
\mathrm{DNF}=\frac{\text { delay neutrons }}{(\text { prompt neutrons }+ \text { delay neutrons })}
$$

\section{Results and Discussion}

From Table 1, it's realized that the inner irradiation recorded highest average flux in all the three energy spectrum regions, and then followed by the outer inner irradiation channel. This may be due to the fact that, these channels are in contact with the annulus beryllium reflector. Beryllium has the ability to undergo $(n, 2 n)$ and $(\gamma, n)$ reactions, these reactions contribute to the delay neutron flux. From Figure 1, it can be observed that the inner irradiation channel in embedded in the beryllium reflector, hence most of these delay neutrons produced from the reflector ends up in the inner channel. The outer channel has only one part of its circular surface touching the beryllium reflector and also furthest from the core hence its lower flux.

The annulus beryllium reflector recorded a higher thermal flux than the core or the moderator because delay neutrons produce in the beryllium undergo enough collision to reach the thermal region and most of these neutron do not have high energies. This phenomenon manifests in the higher energy spectrum region where the neutron flux in the moderator or core region supersedes that of the beryllium region. The delay neutrons produce from the core is as a result of neutrons released by some fission fragments or daughter nuclides, these neutrons

Table 2. Average delay neutron fluxes of neutron energy ranges in various regions of Ghana's MNSR

\begin{tabular}{|c|c|c|c|c|}
\hline Neutron Energy Regions & Moderator & Annulus Beryllium Reflector & Inner Irradiation Channel & Outer Irradiation Channel \\
\hline $\mathrm{MeV}$ & $\times 10^{7} \mathrm{n} / \mathrm{cm}^{2} \cdot \mathrm{s}$ & $\times 10^{7} \mathrm{n} / \mathrm{cm}^{2} \cdot \mathrm{s}$ & $\times 10^{7} \mathrm{n} / \mathrm{cm}^{2} \cdot \mathrm{s}$ & $\times 10^{7} \mathrm{n} / \mathrm{cm}^{2} \cdot \mathrm{s}$ \\
\hline $\begin{array}{c}\text { Thermal Region } \\
(0-6.25 \mathrm{e}-7)\end{array}$ & $(1.2330 \pm 0.0003)$ & $(5.5268 \pm 0.0019)$ & $(40.1270 \pm 0.7620)$ & $(24.5250 \pm 0.0485)$ \\
\hline $\begin{array}{l}\text { Slowing down Region } \\
\text { (8.21e-1 - 6.94) }\end{array}$ & $(0.3092 \pm 0.0017)$ & $(0.0737 \pm 0.0006)$ & $(1.2050 \pm 0.05010)$ & $(0.4146 \pm 0.0372)$ \\
\hline $\begin{array}{l}\text { Fast Region } \\
(6.96-20)\end{array}$ & $(0.0544 \pm 0.0016)$ & $(0.0113 \pm 0.0005)$ & $(0.2065 \pm 0.03260)$ & $(0.0753 \pm 0.0184)$ \\
\hline
\end{tabular}


Table 3. Average delayed and prompt neutron flux in various regions of Ghana's MNSR in the energy range (0 - 20) MeV.

\begin{tabular}{cccc}
\hline Reactor Core Regions & $\begin{array}{c}\text { Delayed Neutron Flux } \\
\times 10^{6} \mathrm{n} / \mathrm{cm}^{2} \cdot \mathrm{s}\end{array}$ & $\begin{array}{c}\text { Prompt Neutron Flux } \\
\times 10^{8} \mathrm{n} / \mathrm{cm}^{2} \cdot \mathrm{s}\end{array}$ & Delayed Neutron Fraction \\
\hline Moderator & $1.128 \pm 0.006$ & $2.460 \pm 0.008$ & $0.00456 \pm 0.00001$ \\
Annulus Beryllium Reflector & $0.517 \pm 0.003$ & $1.135 \pm 0.004$ & $0.00453 \pm 0.00001$ \\
Inner Irradiation Channel & $13.610 \pm 0.562$ & $5.900 \pm 0.118$ & $0.02255 \pm 0.00048$ \\
Outer Irradiation Channel & $4.878 \pm 0.408$ & $2.108 \pm 0.042$ & $0.02262 \pm 0.00144$ \\
\hline
\end{tabular}

are of much higher energy than those produced in the beryllium reflector.

Since delay neutrons are born at relatively low energies, the delay neutron flux tends to peak in the thermal region and significantly reduce as the neutron energies increase towards the fast region.

The delay neutron fractions (DNF) of the various core regions were calculated using Equation (1). From Table $\mathbf{1}$, it is observed that delayed neutrons forms a significant amount of the neutron flux in the inner and outer irradiation channel. The delayed neutrons produced from the beryllium reflector ends up in these regions. The overall delayed neutron fraction of Ghana's MNSR was computed as follows:

Average delayed neutron fractions of the core

$$
\begin{gathered}
=\frac{\text { tatal delayed neutron fraction of coreregions }}{\text { number of core regions }} \\
\mathrm{DNF}=\frac{0.05426 \pm 0.00194}{4}=0.01357 \pm 0.00049
\end{gathered}
$$

For light water reactors using low enriched uranium fuel, the average delayed neutron fraction can change from 0.0070 to 0.0055 as uranium-235 is burned out and plutonium-239 is produced from uranium-238 [8]. The Ghana miniature neutron source reactor is also a light water reactor with $90.2 \%$ uranium-235 enrichment. In view of this high enriched core and the beryllium reflector, the delayed neutron fraction is higher than that of low enriched core

\section{Conclusions}

Delayed neutrons play a great role to the safety of the operation of a nuclear reactor. The Ghana miniature neutron source reactor core has delayed neutron fraction of $0.01357 \pm 0.00049$, showing the contribution of delay neutrons to the total flux contribution of the reactor core. The inner irradiation channel, which has the highest thermal flux, has a significant part of its flux from delayed neutrons, the same applies to the outer irradiation channel. The presence of beryllium around the fuel contributed significantly to the delayed neutron flux in these channels. Most of the delayed neutron flux were thermal neutrons and hence helps to sustain the fission chain reaction. This property of beryllium to act as a reflector and also produce delayed neutron through $(n, 2 n)$ and $(\gamma, n)$ reactions makes it good material for power reactor design where less enrichment will be needed yet long burn-ups will be achieved. Beryllium can also help in the neutron economy of other research reactors to maximize neutron flux which can be harnessed for scientific work, such as in neutron activation analysis (NAA).

\section{References}

[1] J. R. Lamarsh and A. J. Baratta, "Introduction to Nuclear Engineering,” 3rd Edition, Upper Saddle River, 2001, pp. 300-350

[2] E. H. K. Akaho, B. T. Maakuu and M. K. Qazi, “Comparison for Some Measured and Calculated Nuclear Parameters for Ghana Research Reactor-1 Core,” Journal of Applied Science and Technology, Vol. 5, No. 1-2, 2000, pp. 25-31.

[3] G. Kennedy, J. St. Pierre, K. Wang, Y. Zang, J. Preston, C. Grant and M. Vutchkov, "Activation Constant for SLOWPLOKE and MNS Reactors Calculated from the Neutron Spectrum and K0 and Q0 Values," Journal of Radioanalytical Chemistry, Vol. 245, No. 1, 2000, p. 167. doi:10.1023/A:1006749820638

[4] T. Su-De, "Multi-Elemental Analysis of Chinese Biological Standard Reference Materials by Monostandard Instrumental Neutron Activation Analysis," Journal of Radioanalytical Nuclear Chemistry, Vol. 81, No. 2, 1984, p. 81.

[5] I. M. Umar, "The Potentials of MNSR in the Socioeconomic Development of Nigeria,” ICENSIAEA Small Research Reactor Workshop, University of West Indies, Mona, 2003.

[6] S. Anim-Sampong, B. T. Maakuu, E. H. K. Akaho, A. Andam, J. J. R. Liaw and J. E. Matos, "Progress in the Neutronic Core Conversion (HEU-LEU) Analysis of Ghana Research Reactor-1," International RERTR Meeting, Santiago, 23-27 October 2006, pp. 6-7.

[7] S. Anim-Sampong, "Progress Report," ANL RERTR Visiting Scholar (Research Fellow) ANL, 2006.

[8] www.tpub.com/content/doe/h1019v2/css/h1019v2_104.ht $\mathrm{m}$

[9] United Nations International Nuclear Fuel Cycle Evaluation (INFCE), Report of INFCE Working Group 8: Ad- 
vanced Fuel Cycle and Reactor Concepts, Vienna, 25-27 February 1980, p. 17.

[10] G. I. Balogun, S. A. Jonah, Y. A. Ahmed and N. Sa'adu, "Results of on-Site Zero-Power and Criticality Experiments for the Nigerian Research Reactor-1,” Internal Report CERT/NIRR-1/ZP/01, 2004.

[11] E. H. K. Akaho, B. T. Maakuu, S. Anim-Sampong, G. Emi-Reynolds, H. O. Boadu, E. K. Osae, B. S. Akoto and D. N. A. Dodoo-Amoo, "Reactor-1 Final Safety Analysis Report,” Ghana Research, 2003.
[12] S. Anim-Sampong, et al., "Progress in the Neutronic Core Conversion (HEU-LEU) Analysis of Ghana Research Reactor-1," 28th International Meeting on Reduced Enrichment for Research and Test Reactors (RERTR-2007), Cape Town, October 29-November 2, 2007.

[13] S. Anim-Sampong, E. H. K. Akaho, J. K. Gbadago, A. Adam, "Neutronic Analysis for Conversion of the GHARR-1 Facility using Monte Carlo Methods and UO2 LEU Fuel," RRFM and Meeting of the IGORR, Centre de Congres, Lyon, 11-15 March, 2007. 